\title{
The accuracy of presepsin (sCD14-ST) for the diagnosis of sepsis in adults: a meta-analysis
}

\author{
Xin Zhang ${ }^{1 \dagger}$, Dan Liu ${ }^{1,3,4 \dagger}$, You-Ning Liu', Rui Wang ${ }^{2}$ and Li-Xin Xie ${ }^{1 *}$
}

\begin{abstract}
Introduction: The early diagnosis of sepsis remains a challenge. Recently, soluble cluster of differentiation 14 subtype (sCD14-ST), also known as presepsin, has been identified as a potential biomarker of sepsis. We performed a meta-analysis to assess the diagnostic accuracy of presepsin for sepsis in patients with systemic inflammation.

Methods: We systematically searched the PubMed, Embase, Web of Knowledge and Cochrane databases. Studies were included if they assessed the diagnostic accuracy of presepsin for sepsis in adult patients with systemic inflammatory response syndrome (SIRS). Furthermore, a $2 \times 2$ contingency table was constructed based on these results. Two authors independently judged the studies and extracted the data. The diagnostic accuracy of presepsin in sepsis was calculated using a bivariate meta-analysis model. The Q-test and $P$ index were used to test the heterogeneity.

Results: Eight studies involving a total of 1,815 patients were included in the present study. The pooled sensitivity, specificity, diagnostic odds ratio, positive likelihood ratio and negative likelihood ratio were 0.86 (95\% Cl: 0.79-0.91), 0.78 (95 \% Cl: 0.68-0.85), 22 (95\% Cl: 10-48), 3.8 (95\% Cl: 2.6-5.7), and 0.18 (95\% Cl: 0.11-0.28), respectively. The area under the summary receiver operator characteristic curve was 0.89 (95\% Cl: 0.86-0.92). Meta-regression analysis revealed that consecutive patient selection, sample size and setting significantly accounted for the heterogeneity of sensitivity.

Conclusions: Our findings suggest that presepsin exhibits very good diagnostic accuracy ( $A U C=0.89$ ) for the diagnosis for sepsis. Nevertheless, an overall assessment of all the clinical indexes for sepsis diagnosis and continual re-evaluation of presepsin during the course of the disease are needed.
\end{abstract}

\section{Introduction}

Sepsis is a life-threatening condition that arises when the body's response to an infection injures its own tissues and organs [1]. Despite advances in antibiotic therapy and modern life support, the fatality rate of patients with sepsis has remained as high as $30-60 \%[2,3]$. Delays in the initiation of antimicrobial treatment are associated with worse prognosis $[4,5]$, which highlights the importance of timely diagnosis to reduce the morbidity and mortality of sepsis patients.

Currently, the diagnosis of sepsis is based on the presence of systemic inflammatory response syndrome (SIRS) criteria in the presence of a known infection.

\footnotetext{
*Correspondence: ricu301@126.com

${ }^{\dagger}$ Equal contributors

'Department of Pulmonary \& Critical Care Medicine, General Hospital of Chinese People's Liberation Army, 28 Fuxing Road, Beijing 100853, PR China Full list of author information is available at the end of the article
}

However, non-infectious SIRS associated with acute tissue injury and innate immune activation can induce clinical syndromes analogous to sepsis, including multiple trauma, pancreatitis, burns, and autoimmune diseases. Blood culture is the gold standard for identifying infectious conditions, but this approach is of limited use for the early detection of a bloodstream infection due to the duration of time required to obtain positive cultures, exclude specimen contamination and identify colonization [6]. Biomarkers, which were recently introduced among the inflammatory variables in the diagnostic criteria for sepsis, could contribute to the prompt identification of sepsis. However, no ideal biomarker has yet been identified with sufficient clinical sensitivity or specificity for the diagnosis of sepsis [7]. An ideal biomarker is still needed to accurately differentiate sepsis from non-infectious SIRS in a timely and economic manner.

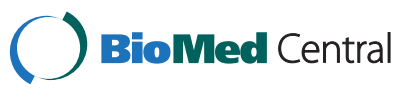

(c) 2015 Zhang et al. Open Access This article is distributed under the terms of the Creative Commons Attribution 4.0 International License (http://creativecommons.org/licenses/by/4.0/), which permits unrestricted use, distribution, and reproduction in any medium, provided you give appropriate credit to the original author(s) and the source, provide a link to the Creative Commons license, and indicate if changes were made. The Creative Commons Public Domain Dedication waiver (http://creativecommons.org/publicdomain/zero/1.0/) applies to the data made available in this article, unless otherwise stated. 
Cluster of differentiation 14 (CD14) is a multifunctional glycoprotein expressed mainly on the membrane surface of monocytes/macrophages. CD14 is also minimally distributed on the cell surface of neutrophils (mCD14) and serves as a specific receptor for complexes of lipopolysaccharides (LPSs) and LPS-binding proteins (LPBs). The LPS-LBP-CD14 complex is released into the circulation by shedding CD14 from the cell membrane, yielding soluble CD14 (sCD14), which is also directly secreted by hepatocytes $[8,9]$. During inflammation, plasma protease activity generates (sCD14) fragments. The 64-amino acid $\mathrm{N}$-terminal fragments constitute the sCD14 subtype (sCD14-ST), which has recently been renamed presepsin [10]. Presepsin has recently been reported to increase in response to the severity of bacterial infections. The plasma levels of presepsin specifically increase during sepsis, and less intensively, during SIRS. An increasing number of studies have demonstrated the ability of presepsin to serve as a valuable marker in sepsis diagnosis [11-18]. In light of the above findings, we aimed to assess the value of presepsin for the diagnosis of sepsis by performing metaanalysis of relevant studies of diagnostic test accuracy. To the best of our knowledge, this approach has not been previously performed.

\section{Methods}

\section{Search strategy and selection criteria}

We systematically searched studies in the PubMed, Embase, Web of Knowledge and the Cochrane Library databases. The search terms were as follows: ("presepsin" or "soluble CD14 subtype" or "sCD14-ST") AND (sepsis OR "bacterial infection" OR "systemic inflammatory response syndrome" OR "SIRS"). No language restriction or publication date restrictions were applied. We further reviewed the reference lists of the selected articles to obtain potentially relevant articles.

Eligible studies were required to include a well-defined reference standard for the patients involved. For the included studies, the sepsis group was defined according to the criteria of the American College of Chest Physicians/Society of Critical Care Medicine [19, 20], whereas the other patients were required to meet the criteria for SIRS. Additionally, the studies included data on the diagnostic accuracy of presepsin for sepsis in adult patients ( $>18$ years old) with SIRS. Furthermore, a $2 \times 2$ contingency table was constructed based on the results. For studies providing multiple presepsin cutoff values for diagnostic accuracy, the data providing the maximum overall accuracy were chosen. For studies that evaluated the diagnostic accuracy of presepsin levels at multiple time points, we chose the data based on the initial presepsin level. Reviews, letters, commentaries, correspondence, case reports, conference abstracts, expert opinions, editorials and animal experiments were excluded.
Articles involving paediatric patients were also excluded. Two investigators (Zhang X and Liu D) independently performed the search strategy and evaluated the studies. Any disagreement was resolved by a third opinion.

\section{Data extraction and quality assessment}

The following descriptive data were extracted from the original studies: the name of the first author, publication year, country of origin, study design, clinical setting, severity of sepsis, control patients, sample size, prevalence of sepsis, the percentage of male patients, average age, cutoff points, and the true positive (TP), false positive (FP), false negative (FN), and true negative (TN) rates, sensitivity (SEN) and specificity (SPE) of the data. We contacted the corresponding authors if the necessary data were not presented or required clarification. We used a revised tool for the Quality Assessment of Diagnostic Accuracy Studies (QUADAS) checklist [21] to assess the quality of the included studies. Furthermore, the studies were grouped according to Sackett and Haynes' classification of diagnostic studies [22]. Utilizing this classification, phase 1 studies were those that compared the difference in test results between patients with the target disorder and healthy individuals. Phase 2 studies were those that examined how the index test differentiated between patients with and without the target disorder. Phase 3 studies were those that assessed the test's real-life performance in patients in whom the disorder was suspected.

\section{Statistical analysis}

All statistical analyses were performed using the MIDAS module for STATA software, version 12.0 (Stata Corporation, College Station, TX, USA). P values less than 0.05 were considered statistically significant. A bivariate random effects regression model [23] was used to calculate the pooled SEN, SPE, diagnostic odds ratio (DOR), positive likelihood ratio (PLR), and negative likelihood ratio (NLR). We also constructed a summary receiver operator characteristic (SROC) curve by plotting the individual and summary points of SEN and SPE to assess overall diagnostic accuracy [24]. Fagan's nomogram was used to calculate the post-test probability (PTP) [25]. The $Q$ test and $I^{2}$ index were calculated to assess between-study heterogeneity $[26,27] . I^{2}$ greater than $50 \%$ was considered sustainable heterogeneity among studies.

In addition to the proportion of heterogeneity that was likely due to the threshold effect, univariate metaregression analysis and subgroup analysis were performed to explore the sources of potential heterogeneity in SEN and SPE. The covariates included the following: prevalence (prevalence $<50 \%$ or $\geq 50 \%$ ), sample size (sample size $<100$ or $\geq 100$ ), blinding (whether sepsis 
patients were diagnosed without knowledge of the results of the presepsin level), setting (whether the study was performed in the emergency department (ED) or intensive care unit (ICU)), study design (whether patients were recruited consecutively), and comorbidities (whether the studies excluded patients who had comorbidities that were likely to influence presepsin levels). Subgroup analysis was restricted to ED patients. Publication bias was tested by Deek's funnel plot.

\section{Results}

Our database search retrieved 185 articles, 172 of which were eliminated for various reasons based on the title and abstract, leaving 13 studies that were scrutinized in a full-text review. Among the 13 studies, one study investigated the prognostic value of presepsin in sepsis, one could not be used to reconstruct the $2 \times 2$ table, and three were performed using an ineligible design (i.e., they evaluated the diagnostic accuracy of presepsin based on samples that were collected at multiple time points but not every individual sample). In total, eight studies fulfilled our eligibility criteria and were included in the final analysis (Fig. 1). We did not identify any additional relevant articles in the bibliographies of the original articles.

\section{Characteristics of the included studies}

The included studies were published between 2012 and 2014. Four studies $[11,14,16,18]$ were conducted in Europe, and four $[12,13,15,17]$ were conducted in

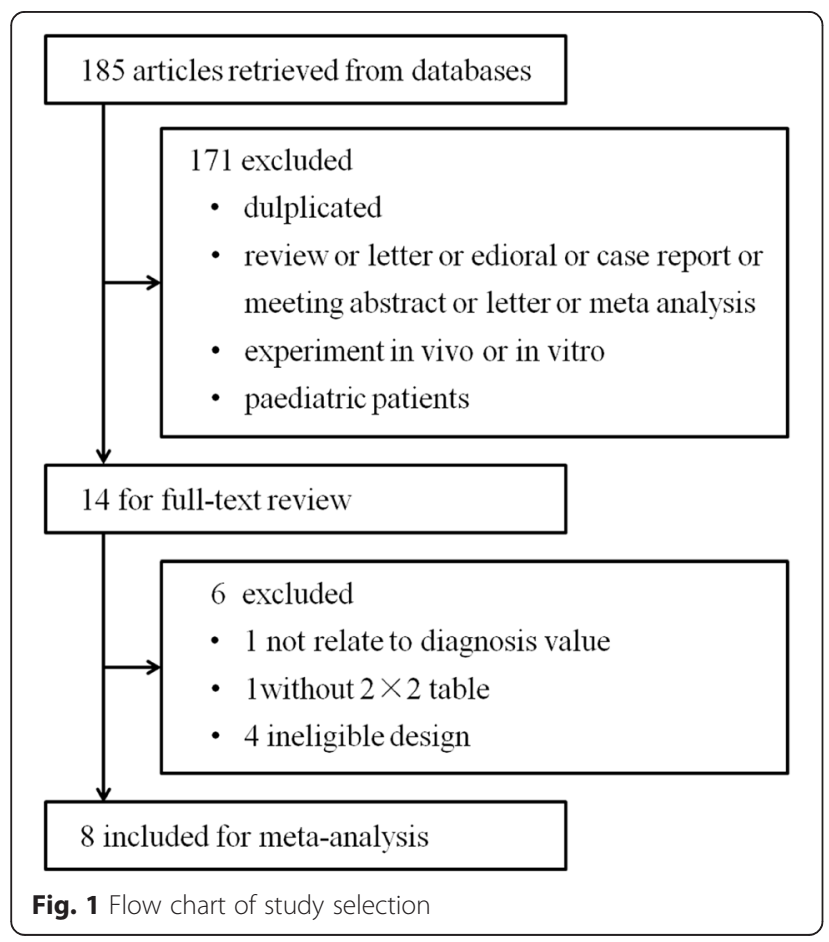

Asia. A total of 1,815 patients were included, and SIRS criteria were fulfilled in 1,690 patients, including 1,165 patients with septis and 525 patients with non-infectious SIRS. Four studies recruited a group of well-matched (by age and sex) patients without SIRS (60 patients in Behnes [11], 25 patients in Kweon [12], 100 patients in Liu [13], and 70 patients in Vodnik [14]) as controls, and two studies $[12,13]$ included the control patients as well as the SIRS patients in the non-sepsis group in a $2 \times 2$ contingency table when analysing the diagnostic accuracy of presepsin for sepsis. The mean age of the patients varied between 54.4 and 79.42 years, and the proportion of male patients ranged from 50.0 to 66.3 . The prevalence of sepsis varied from $16.4 \%$ to $79.2 \%$. The most frequent source of sepsis was pulmonary infection. Six studies [12-14, 16-18] were performed in the ED, one [11] in the ICU, and one [15] in the ED and ICU. All included studies recruited a mix of medical and surgical patients. One study [12] excluded patients with comorbidities (e.g., chronic renal failure or a history of resuscitation and trauma) that could influence presepsin levels. Presepsin levels were measured with a chemiluminescent immunoassay on a PATHFAST immunoanalyzer $[28,29]$ in all studies. The test threshold ranged from 317 to $729 \mathrm{pg} / \mathrm{ml}$. Details of all eight studies are presented in Table 1 . The optimal cutoff point was retrospectively determined based on the ROC curve. The mean cutoff for presepsin in the included studies was $560 \mathrm{pg} / \mathrm{ml}$ (IQR 317-729).

\section{Study quality and publication bias}

Studies were grouped according to Sackett and Haynes' classification for diagnostic studies: two were phase 2 studies (group 1) [12, 13], and six were phase 3 studies (group 2) [11, 14-18]. All studies included a prospective cohort. Two $[15,18]$ studies were multicentre trials. One study [13] consecutively enrolled patients. Two studies $[12,13]$ excluded patients with comorbidities that could influence presepsin levels. The QUADAS checklists are presented in Fig. 2. On average the overall QUADAS scores of all studies met 10 of the 14 criteria, which suggests that the studies were of high quality. Deek's funnel plot is presented in Fig. 3. No significant publication bias was observed $(p=0.31)$.

\section{Data synthesis and meta-analysis}

The pooled SEN and SPE were 0.86 (95 \% CI: 0.79, 0.91) and 0.78 (95\% CI: 0.68, 0.85), respectively (Fig. 4). The PLR and NLR were 3.8 (95\% CI: 2.6, 5.7) and 0.18 (95\% CI: $0.11,0.28)$, respectively (Fig. 5). The DOR was 22 (95\% CI: $10,48)$. The area under the SROC curve was 0.89 (95\% CI: 0.86, 0.92) (Fig. 6). Figure 7 presents Fagan's nomogram for likelihood ratios, and the results indicate that the use of presepsin in the detection of sepsis increased the post- 
Table 1 Characteristics of included studies

\begin{tabular}{|c|c|c|c|c|c|c|c|c|c|c|c|c|c|c|c|c|c|}
\hline Author & Year & Country & Study design & Clinical setting & Severity of sepsis & Control patients & $\begin{array}{l}\text { Sample } \\
\text { size }(n)\end{array}$ & $\begin{array}{l}\text { Prevalence } \\
(\%)\end{array}$ & $\begin{array}{l}\text { Male } \\
(\%)\end{array}$ & $\begin{array}{l}\text { Mean/median } \\
\text { age (years) }\end{array}$ & $\begin{array}{l}\text { Cutoff } \\
(\mathrm{pg} / \mathrm{ml})\end{array}$ & TP & FP & $\mathrm{FN}$ & TN & SEN & SPE \\
\hline \multicolumn{18}{|l|}{ Group 1 studies } \\
\hline Kweon [12] & 2014 & Korea & PR & ED & $\begin{array}{l}\text { Sepsis, severe sepsis } \\
\text { and septic shock }\end{array}$ & $\begin{array}{l}25 \text { patients } \\
\text { without SIRS }\end{array}$ & 118 & 61.9 & 50 & 61.19 (20-91) & 430 & 64 & 8 & 9 & 37 & 87.7 & 82.2 \\
\hline Liu [13] & 2013 & China & $P R+C R$ & ED & $\begin{array}{l}\text { Sepsis, severe sepsis } \\
\text { and septic shock }\end{array}$ & $\begin{array}{l}100 \text { healthy } \\
\text { controls }\end{array}$ & 859 & 79.2 & 66.3 & 79.42 (58-78) & 317 & 481 & 40 & 199 & 239 & 70.8 & 85.8 \\
\hline \multicolumn{18}{|l|}{ Group 2 studies } \\
\hline Vodnik [14] & 2013 & Serbia & PR & ED & $\begin{array}{l}\text { Sepsis, severe sepsis } \\
\text { and septic shock }\end{array}$ & $\begin{array}{l}70 \text { healthy } \\
\text { controls }\end{array}$ & 60 & 50 & 58.3 & $54.4 \pm 15.5$ & 630 & 30 & 2 & 0 & 28 & 100 & 93 \\
\hline Endo [15] & 2012 & Japan & MPR & $\mathrm{ED}$ and ICU & NA & No & 185 & 62.1 & 59.5 & 71.8 (17-98) & 600 & 101 & 13 & 14 & 57 & 87.8 & 81.4 \\
\hline $\begin{array}{l}\text { de Guadiana } \\
\text { Romualdo [16] }\end{array}$ & 2014 & Spain & PR & ED & NA & No & 226 & 16.4 & 58.4 & $67 \pm 26$ & 729 & 30 & 70 & 7 & 119 & 81.1 & 63 \\
\hline Ishikura [17] & 2014 & Japan & PR & ED & $\begin{array}{l}\text { Sepsis, severe sepsis } \\
\text { and septic shock }\end{array}$ & No & 82 & 52.4 & 53.7 & $67.2 \pm 17.3$ & 647 & 39 & 9 & 4 & 30 & 93 & 76.3 \\
\hline Ulla [18] & 2013 & Italy & MPR & ED & $\begin{array}{l}\text { Sepsis and septic } \\
\text { shock }\end{array}$ & No & 189 & 56.1 & 61.4 & 64.4 (19-99) & 600 & 84 & 32 & 22 & 51 & 78.95 & 61.9 \\
\hline Behnes [11] & 2014 & Germany & $P R$ & $\mathrm{ICU}$ & $\begin{array}{l}\text { Severe sepsis and } \\
\text { septic shock }\end{array}$ & $\begin{array}{l}60 \text { patients } \\
\text { without SIRS }\end{array}$ & 96 & 84.4 & 63.1 & 65.89 (20-88) & 530 & 73 & 6 & 8 & 9 & 90 & 60 \\
\hline
\end{tabular}

$P R$ prospective recruitment, $M P R$ multicentre prospective recruitment, $C R$ consecutive recruitment, $E D$ emergency department, $N A$ not available $T P$ true positive, $F P$ false positive, $T N$ true negative, $F N$ false negative, SIRS systemic respiratory response syndrome, SEN sensitivity, SPE specificity 


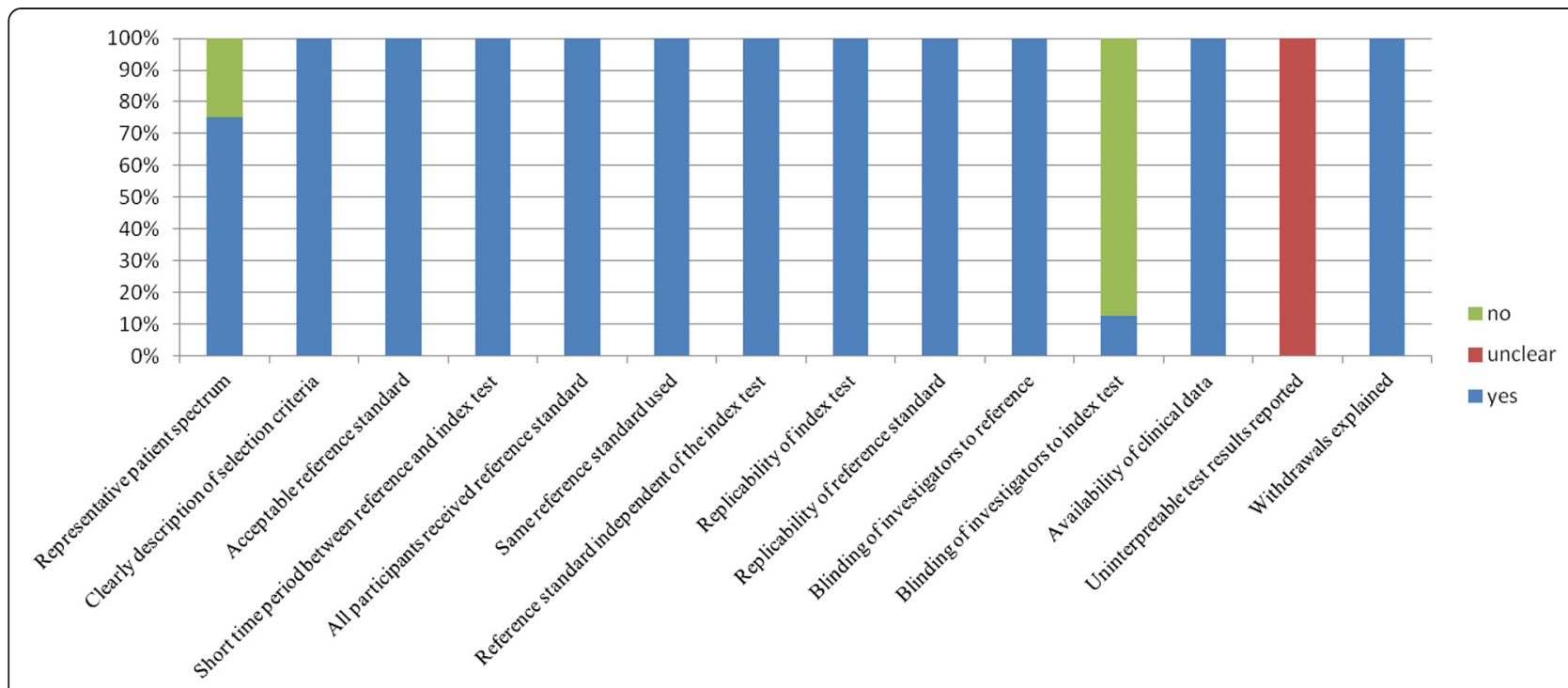

Fig. 2 Proportion of Quality Assessment of Diagnostic Accuracy Studies (QUADAS) tool criteria fulfilled by the included studies

probability to $48 \%$ when the results were positive and reduced the post-probability to $4 \%$ when the results were negative. The mean cutoff for presepsin in the included studies was $560 \mathrm{pg} / \mathrm{ml}$ (IQR 317-729).

Six studies comprising 838 patients were included in group 2 studies (Table 1). We performed a subgroup analysis restricted to this group because the studies were restricted to patients who were most likely to be encountered by clinicians and who were more informative for routine clinical practice. The pooled SEN and SPE were 0.89 (95\% CI: $0.81,0.94)$ and 0.75 (95 \% CI: 0.64, 0.84), respectively. The PLR and NLR were 3.6 (95\% CI: 2.2, 5.8) and 0.15 (95\% CI: 0.08, 0.29), respectively. The DOR was 24 (95\% CI: 8,71$)$. The area under the SROC curve was 0.90 (95\% CI: $0.87,0.92)$. The $I^{2}$ test results for the pooled SEN and SPE were $68.22 \%$ and $84.90 \%$, respectively.

There was substantial heterogeneity among the studies. The $I^{2}$ test results for the pooled SEN and SPE were

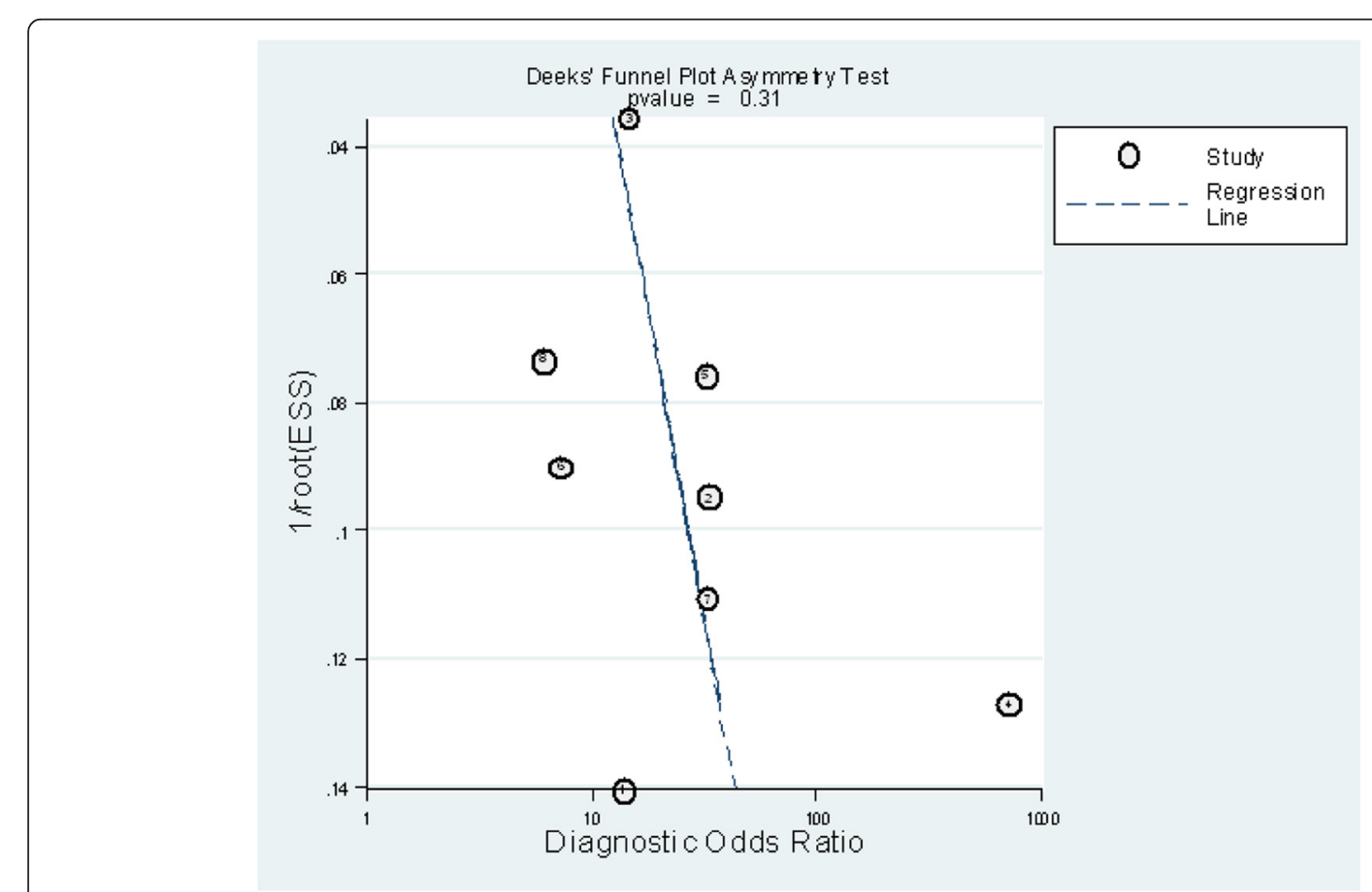

Fig. 3 Deek's funnel plot asymmetry test for publication bias. No publication bias was detected $(p=0.31)$ 


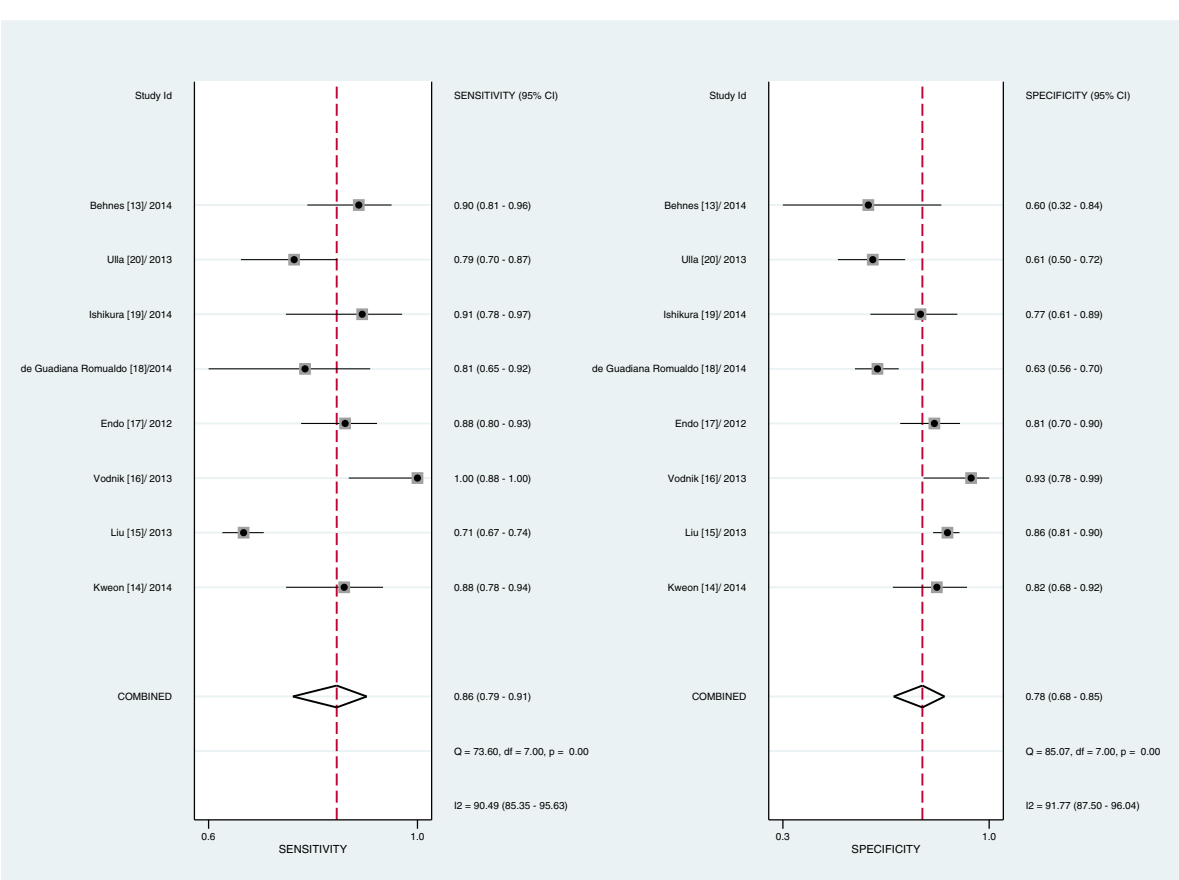

Fig. 4 Forrest plot of the sensitivity and specificity of presepsin for the diagnosis of sepsis

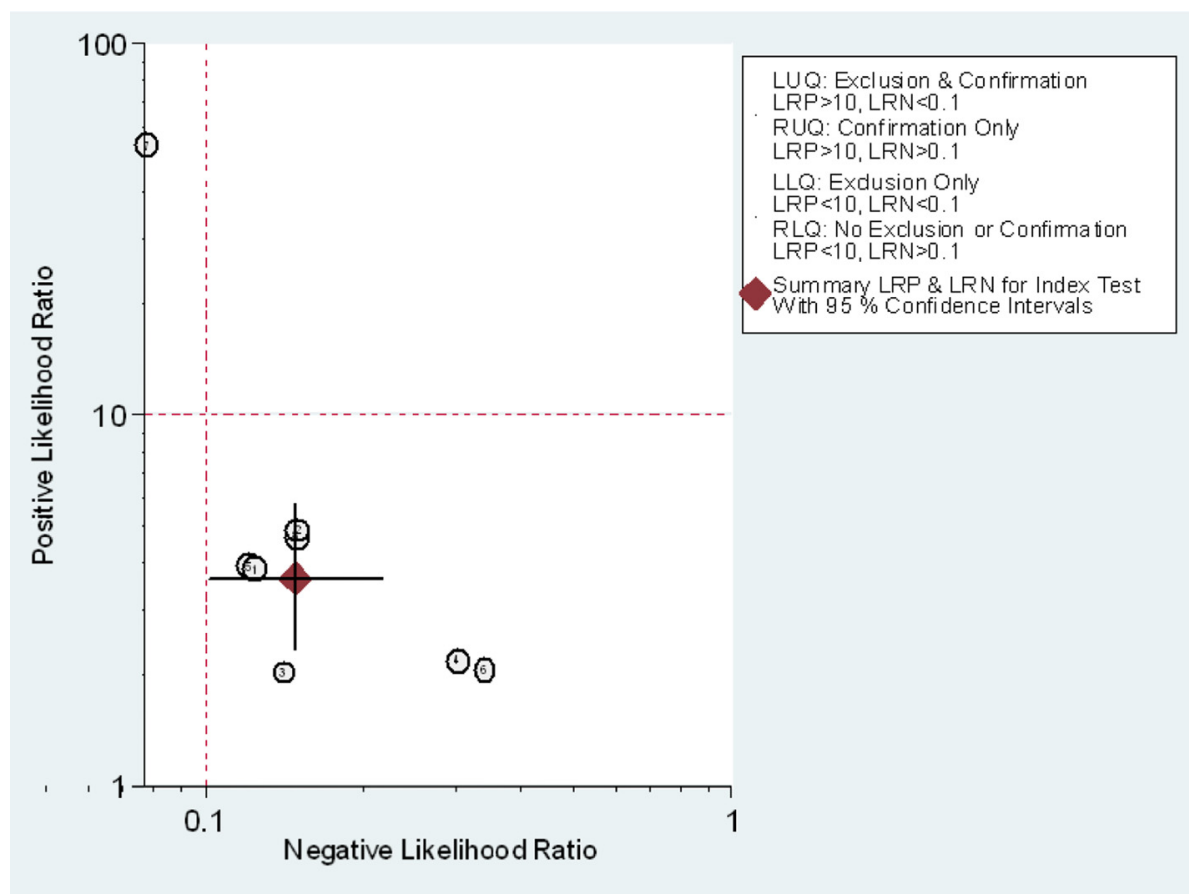

Fig. 5 Positive and negative likelihood ratios. The positive likelihood ratio and negative likelihood ratio were 3.8 (95 \% Cl: $2.6,5.7), 0.18$ (95 \% Cl: $0.11,0.28)$, respectively LUQ left upper quadrant, RUQ right upper quadrant, LLQ left lower quadrant, RLQ right lower quadrant, LRP likelihood ratio positive, LRN likelihood ratio negative 


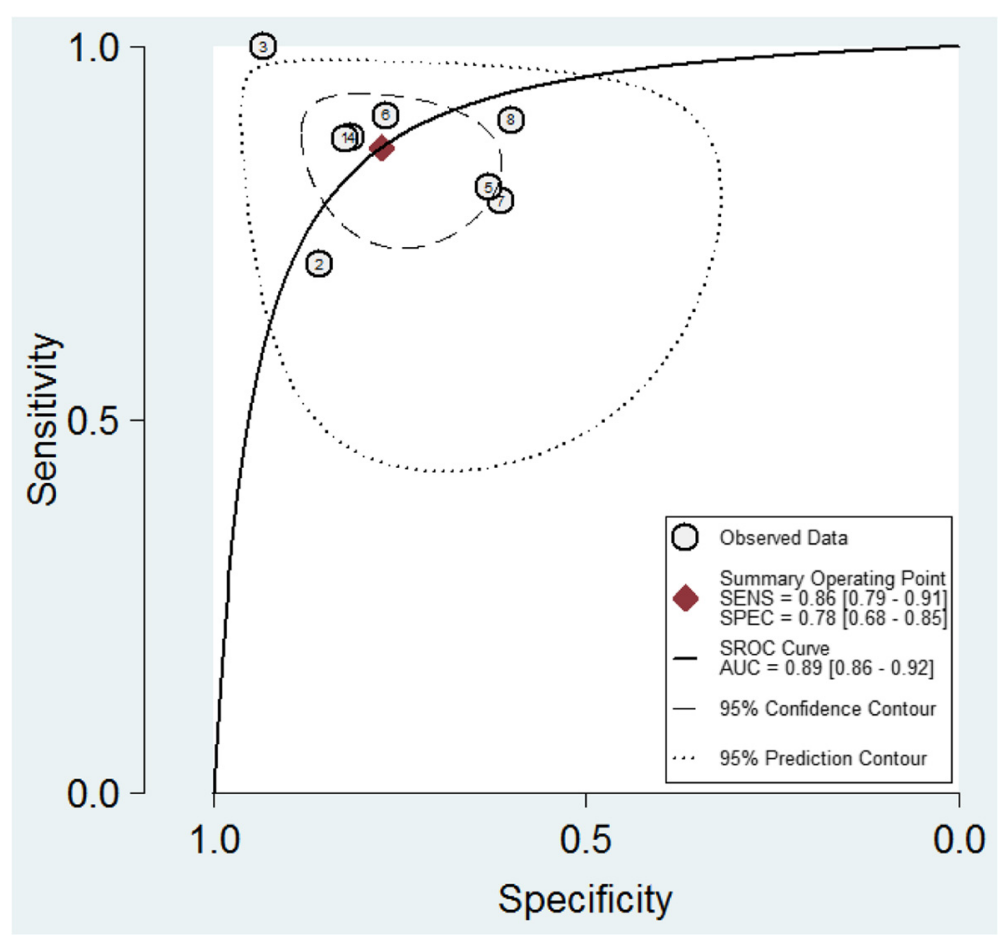

Fig. 6 Summary receiver operating characteristic graph of included studies. SEN sensitivity, SPE specificity

$90.49 \%$ and $91.77 \%$, respectively. The overall $I^{2}$ values for the bivariate model were $94 \%$ (95 \% CI: 85, 99). The proportion of heterogeneity likely caused by the threshold effect was small (0.07), whereas the variations in SEN and SPE were related to differences in the cutoff points for presepsin that were used in the included studies.

Univariate meta-regression analysis and subgroup analysis were performed to explore the sources of potential heterogeneity in SEN and SPE. Patient blinding, prevalence, setting, consecutive patient recruitment, and sample size were used as covariates. Meta-regression revealed that consecutive recruitment, sample size, and setting significantly accounted for the heterogeneity of sensitivity (Fig. 8). The subgroup analysis restricted to ED patients revealed that the pooled SEN and SPE were 0.85 (95\% CI: $0.77,0.92)$ and 0.78 (95\% CI: 0.69, 0.88), respectively.

\section{Discussion}

Sepsis is a common problem in critically ill patients. Early diagnosis and early treatment are essential for the clinical course and the outcome of patients with sepsis. Given that a large proportion of critically ill patients exhibit SIRS, the ability to accurately distinguish between SIRS and sepsis, which is defined as SIRS as a result of bacterial infection, has become one of the holy grails of critical care medicine.

Currently, no recommendation can be given for the use of biomarkers to differentiate sepsis from non- infectious SIRS [30]. Many biomarkers, particularly procalcitonin (PCT) and C-reactive protein (CRP), are widely used to identify sepsis in current clinical practice $[31,32]$. In comparison with CRP, PCT seems to be a better marker to differentiate sepsis from non-infectious SIRS. However, two published meta-analyses concluded that the SEN and SPE of PCT varies in the diagnosis of sepsis, leading to questions of the ability of PCT to distinguish sepsis from SIRS [33, 34]. Presepsin was recently identified as a molecule involved in the inflammatory response [35] and represents a promising diagnostic biomarker with high SEN and SPE.

The primary finding of our meta-analysis is that presepsin exhibits very good diagnostic accuracy for distinguishing patients with sepsis from those with systemic inflammatory disease. Specifically, in our primary analysis involving all studies that evaluated presepsin in adults with and without sepsis, the area under the SROC curve was 0.89 , which was greater than the results of published meta-analyses of the use of PCT and soluble triggering receptor expressed on myeloid cells-1 (sTREM-1) for the diagnosis of sepsis $[33,34,36]$. The pooled SEN and SPE were $86 \%$ and $78 \%$, respectively. To our knowledge, presepsin exhibited the highest sensitivity among the proposed biomarkers in differentiating sepsis form other non-infectious SIRS. To date, no biomarkers have exhibited sufficient (greater than $90 \%$ ) sensitivity to distinguish sepsis from SIRS in these critically ill adult patients. 


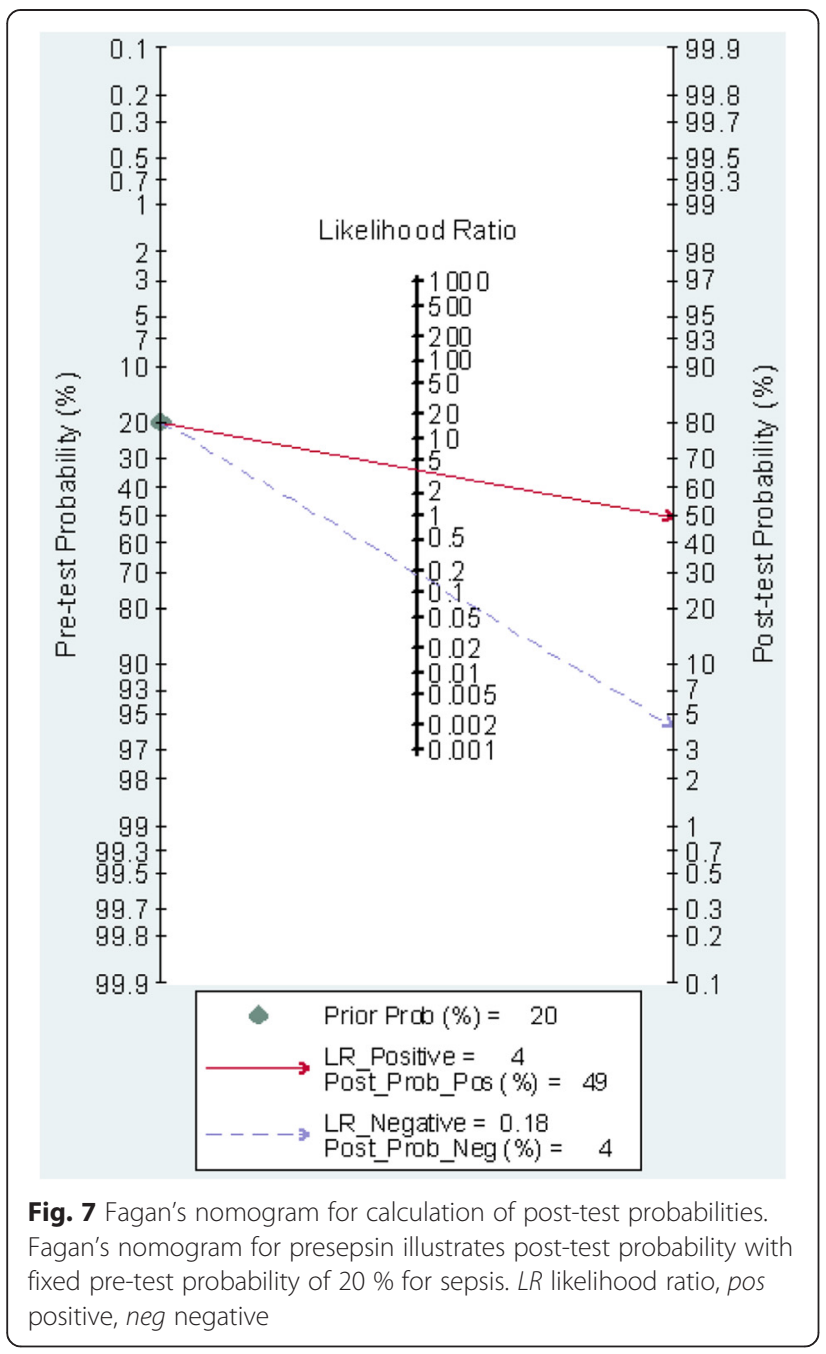

The rescue principles indicate that the infection foci of patients with sepsis should be detected within 6 hours, followed by antibiotic treatment within 1 hour after the diagnosis of sepsis [37]. Generally, PCT increases 4 hours after infection, slowly reaching a plateau at 8-24 hours and peaking one day after infection. Compared with PCT, presepsin increases at 2 hours post-infection in the cecal ligation and puncture (CLP) sepsis model and peaks at 3 hours [29]. Presepsin can be detected in the early stage of infection using rapid dosage methods based on chemiluminescence enzyme immunoassay, which are available and permit automated measurements in 1.5 hours [38].

The exclusion of reviews, letters, commentaries, correspondence, case reports, conference abstracts, expert opinions, editorials and reports of animal experiments may have contributed to publication bias. However, we tested for this, and no significant publication bias was observed in our study (Fig. 3).
Marked statistical heterogeneity was present in all analyses, a fact that must not be overlooked in the interpretation of the above findings. We observed significant heterogeneity in SEN and SPE among the studies analysed. Consecutive patient recruitment, sample size, setting and excluded patients substantially affected the SEN of the diagnosis of sepsis in the meta-regression analysis, and none of the variables affected the SPE of diagnosis of bacterial infection in the meta-regression analysis. According to Sackett and Haynes' classification [22], the index test in group 1 is developed in an ideal situation against a validation set (group 2) in which the performance is tested in a more realistic clinical context. Group 2 studies are the most informative for clinical practice, as they are designed to resemble the real-world setting of routine clinical practice by restriction to patients who are the most likely to be encountered by clinicians. In this meta-analysis, six of the included studies were classified as group 2 studies. Additionally, we performed subgroup analysis restricted to group 2 . The pooled sensitivity, DOR and AUC of the six studies (11, 14-18) were similar to corresponding values in the other studies. In particular, the pooled AUC indicated high diagnostic accuracy (AUC 20.9) [39]. All of the studies included involved a prospective cohort, and two were multicentre studies, underlining the high quality of these studies. The test results obtained by the prospective recruitment (PR) study design method achieved more realistic results than that obtained by the consecutive recruitment $(\mathrm{CR})$ method. Therefore, satisfactory results could be achieved in the future by implementing more prospective studies.

Likelihood ratios and PTPs are also relevant for clinicians, as they provide information on the likelihood of a patient with a positive or negative test actually exhibiting sepsis. In our study, with a hypothetical pretest probability of $20 \%$ and a PLR of 3.8, detecting presepsin for sepsis diagnosis would raise the PTP to $49 \%$, with an NLR of 0.18 . Detecting presepsin reduced the PTP to $4 \%$ (Fig. 7), demonstrating that the application of the presepsin test was advantageous in the diagnosis of sepsis. Additionally, all included studies recruited a mix of medical and surgical patients. Our findings can therefore be generalized to patients from different countries as well as to different admission categories.

Delayed resuscitation is reportedly significantly associated with a high risk of death $[40,41]$, and rapidly initiating the appropriate therapeutic interventions upon the patient's arrival to the ED is critical. Thus, we performed subgroup analysis restricted to ED patients to evaluate the diagnostic accuracy of presepsin. The pooled SEN and SPE were 0.85 (95 \% CI: 0.77, 0.92) and 0.78 (95\% CI: $0.69,0.88)$, respectively, nearly equal to the overall results. 


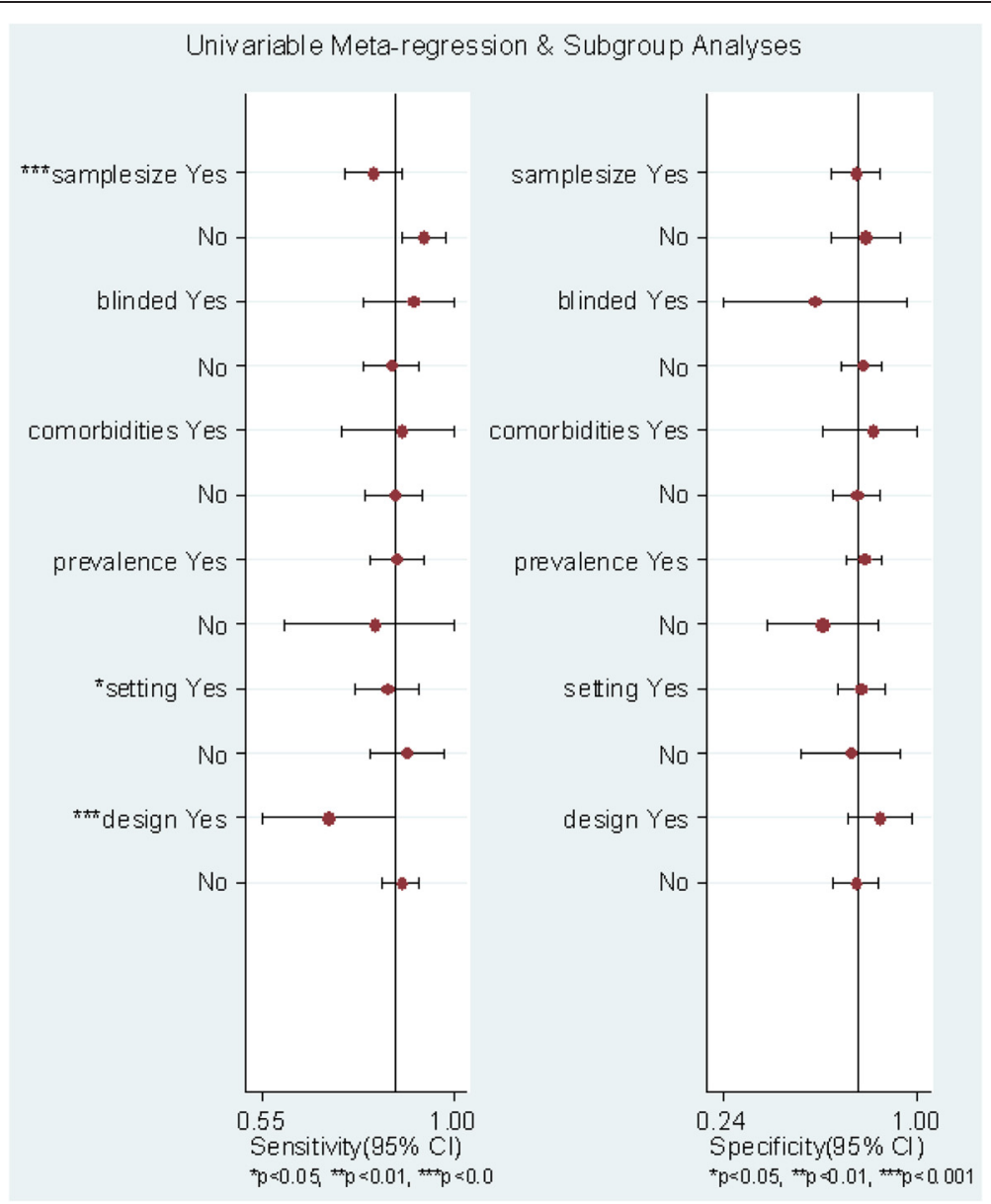

Fig. 8 Univariate meta-regression and subgroup analysis

Several limitations should be considered when interpreting the findings of this meta-analysis. First, despite the extensive literature search, the number of included studies was small; however, the number of patients enrolled was satisfactory $(\mathrm{n}=1,815)$, thereby decreasing type II error. Second, we could not determine the optimized cutoff value because we failed to obtain the raw data to map the ROC curve. In all studies, the optimal cutoff point was retrospectively determined based on the ROC curve. The cutoff points varied greatly among the studies, despite using the same presepsin assay. A reason for this discrepancy may be differences in study design, especially the patient inclusion criteria. Falsely elevated values of presepsin or PCT are observed in conditions of chronic renal failure or a history of resuscitation and trauma. One study [12] excluded patients with these comorbidities, but the others did not. Thus, future research should be designed in consideration of how comorbidities may influence presepsin levels to confirm an optimal cutoff point for clinical use. Third, due to the small number of eligible studies and the lack of necessary data reported in the original publications, we could not specifically analyse patients with different conditions (e.g., different severities of sepsis or different sites of infection) to distinguish the sepsis, nor could we determine the therapeutic decisions in the individual patient. Last, it is possible that presepsin may perform differently in sepsis caused by gram-positive, gram-negative, or fungal pathogens. Hence, both the clinical characteristics of the enrolled patients as well as the local microbiological profile in included studies are likely to affect the value of presepsin in predicting sepsis. However, we were unable to explore this further because the necessary information was largely unavailable in the studies.

\section{Conclusions}

Despite these limitations, our study is the first comprehensive meta-analysis to date that assesses the diagnostic accuracy of presepsin for sepsis in patients with SIRS. The results demonstrated that presepsin may be a reliable biomarker for sepsis because of its good overall diagnostic performance. However, presepsin cannot be recommended as the single definitive test for sepsis diagnosis according to the current data. Clinicians must 
comprehensively evaluate the condition of each patient and should incorporate an overall assessment of the clinical indexes for sepsis diagnosis rather than focusing only on a single biomarker-based approach. Moreover, continuous re-evaluation during the course of the disease is advisable.

\section{Key messages}

- The present study showed that the pooled sensitivity and specificity of presepsin for sepsis diagnosis was 0.86 and 0.78 , respectively. The area under the SROC curve was 0.89

- Presepsin has very good diagnostic accuracy $($ AUC $=0.89)$ for the diagnosis for sepsis in patients with SIRS. However, it cannot be recommended as the single definitive test for sepsis diagnosis according to current data

\section{Abbreviations}

CR: consecutive recruitment; CRP: C-reactive protein; DOR: diagnostic odds ratio; ED: emergency department; FN: false negative; FP: false positive; ICU: intensive care unit; NLR: negative likelihood ratio; PCT: procalcitonin: PLR: positive likelihood ratio; PR: prospective recruitment; PTP: post-test probability; SCD14-ST: soluble CD14-subtype; SEN: sensitivity; SIRS: systemic inflammatory response syndrome; SPE: specificity; TN: true negative; TP: true positive.

\section{Competing interests}

The authors declare that they have no competing interests.

\section{Authors' contributions}

XZ and DL had full access to all of the data in the study and take responsibility for the integrity of the data and the accuracy of the data analysis. Y-NL contributed to conception, design, data interpretation, and manuscript revision for critical intellectual content. RW contributed to the study design, data collection, interpretation, and statistical analysis, and manuscript preparation. L-XX contributed to conception, design, data interpretation, manuscript revision for critical intellectual content, and supervision of the study. All authors read and approved the manuscript.

\section{Author details}

'Department of Pulmonary \& Critical Care Medicine, General Hospital of Chinese People's Liberation Army, 28 Fuxing Road, Beijing 100853, PR China. ${ }^{2}$ Department of Clinical Pharmacology, General Hospital of Chinese People's Liberation Army, 28 Fuxing Road, Beijing 100853, PR China. ${ }^{3}$ Medical School, Nankai University, 94 Weijin Road, Tianjin 300071, PR China. ${ }^{4}$ Department of Respiratory Medicine, Tianjing Medical University General Hospital, Tianjin 300070, PR China.

Received: 13 December 2014 Accepted: 13 August 2015

Published online: 11 September 2015

\section{References}

1. Czura CJ. Merinoff Symposium 2010: sepsis-speaking with one voice. Mol Med. 2011;17:2-3.

2. Linde-Zwirble WT, Angus DC. Severe sepsis epidemiology: sampling, selection, and society. Crit Care. 2004;8:222-6.

3. Dombrovskiy VY, Martin AA, Sunderram J, Paz HL. Rapid increase in hospitalization and mortality rates for severe sepsis in the United States: a trend analysis from 1993 to 2003. Crit Care Med. 2007:35:1244-50.

4. Lodise TP, Mc Kinnon PS, Swiderski L, Rybak MJ. Outcomes analysis of delayed antibiotic treatment for hospital-acquired Staphylococcus aureus bacteremia. Clin Infect Dis. 2003;36:1418-23.

5. Lodise TP, Patel JR, Kwa A, Graves J, Furuno JP, Graffunder E, et al. Predictors of 30-day mortality among patients with Pseudomonas aeruginosa bloodstream infections: impact of delayed appropriate antibiotic selection. Antimicrob Agents Chemother. 2007;51:3510-5.

6. Schuetz $P, A$ min DN, Greenwald JL. Role of procalcitonin in managing adult patients with respiratory tract infections. Chest. 2012;141:1063-73.

7. Sandquist M, Wong HR. Biomarkers of sepsis and their potential value in diagnosis, prognosis and treatment. Expert Rev Clin Immunol. 2014;21:1-8.

8. Grunwald U, Krüger C, Westermann J, Lukowsky A, Ehlers M, Schütt C. An enzyme-linked immunosorbent assay for the quantification of solubilized CD14 in biological fluids. J Immunol Method. 1992;155:225-32.

9. Landmann R, Zimmerli W, Sansano S, Link S, Hahn A, Glauser MP, et al. Increased circulating soluble CD14 is associated with high mortality in gram-negative septic shock. J Infect Dis. 1995;171:639-44.

10. Mussap M, Noto A, Fravega M, Fanos V. Soluble CD14 subtype presepsin (sCD14-ST) and lipopolysaccharide binding protein (LBP) in neonatal sepsis: new clinical and analytical perspectives for two old biomarkers. J Matern Fetal Neonatal Med. 2011;24:12-4.

11. Behnes M, Bertsch T, Lepiorz D, Lang S, Trinkmann F, Brueckmann M, et al. Diagnostic and prognostic utility of soluble CD 14 subtype (presepsin) for severe sepsis and septic shock during the first week of intensive care treatment. Crit Care. 2014;18:507.

12. Kweon OJ, Choi JH, Park SK, Park AJ. Usefulness of presepsin (sCD14 subtype) measurements as a new marker for the diagnosis and prediction of disease severity of sepsis in the Korean population. J Crit Care. 2014;29:965-70.

13. Liu B, Chen YX, Yin Q, Zhao YZ, Li CS. Diagnostic value and prognostic evaluation of Presepsin for sepsis in an emergency department. Crit Care. 2013;17:R244.

14. Vodnik T, Kaljevic G, Tadic T, Majkic-Singh N. Presepsin (sCD14-ST) in preoperative diagnosis of abdominal sepsis. Clin Chem Lab Med. 2013;51:2053-62.

15. Endo S, Suzuki Y, Takahashi G, Shozushima T, Ishikura H, Murai A, et al. Usefulness of presepsin in the diagnosis of sepsis in a multicenter prospective study. J Infect Chemother. 2012;18:891-7.

16. Romualdo LG, Torrella PE, González MV, Sánchez RJ, Holgado AH, Freire AO, et al. Diagnostic accuracy of presepsin (soluble CD14 subtype) for prediction of bacteremia in patients with systemic inflammatory response syndrome in the Emergency Department. Clin Biochem. 2014;47:505-8.

17. Ishikura H, Nishida T, Murai A, Nakamura Y, Irie Y, Tanaka J, et al. New diagnostic strategy for sepsis-induced disseminated intravascular coagulation: a prospective single-center observational study. Crit Care. 2014;18:R19.

18. Ulla M, Pizzolato E, Lucchiari M, Loiacono M, Soardo F, Forno D, et al. Diagnostic and prognostic value of presepsin in the management of sepsis in the emergency department: a multicenter prospective study. Crit Care. 2013;17:R168.

19. Levy MM. SCCM/ESICM/ACCP/ATS/SIS International Sepsis Definitions Conference. Intensive Care Med. 2001;2003:530-8.

20. American College of Chest Physicians/Society of Critical Care Medicine Consensus Conference. Definitions for sepsis and organ failure and guidelines for the use of innovative therapies in sepsis. Crit Care. 1992;20:864-74.

21. Whiting P, Rutjes AW, Reitsma JB, Bossuyt PM, Kleijnen J. Thedevelopment of QUADAS: a tool for the quality assessment ofstudies of diagnostic accuracy included in systematic reviews. BMC Med Res Methodol. 2003;3:25.

22. Sackett DL, Haynes RB. The architecture of diagnostic research. BMJ. 2002:324:539-41.

23. Reitsma JB, Glas AS, Rutjes AW, Scholten RJ, Bossuyt PM, Zwinderman AH. Bivariate analysis of sensitivity and specificity produces informative summary measures in diagnostic reviews. J Clin Epidemiol. 2005;58:982-90.

24. Moses $L E$, Shapiro D, Littenberg B. Combining independent studies of a diagnostic test into a summary ROC curve: data-analytic approaches and some additional considerations. Stat Med. 1993;12:1293-316.

25. Fagan TJ. Letter: Nomogram for Bayes theorem. N Engl J Med. 1975;293:257.

26. Higgins JP, Thompson SG. Quantifying heterogeneity in a meta-analysis. Stat Med. 2002;21:1539-58.

27. Cucherat M, Easy MA. A program for the meta-analysis of clinical trials. Comput Methods Programs Biomed. 1997:53:187-90.

28. Kurihara T, Yanagida A, Yokoi H, Koyata A, Matsuya T, Ogawa J, et al. Evaluation of cardiac assays on a benchtopchemiluminescent enzyme immunoassay analyzer, PATHFAST. Anal Biochem. 2008;375:144-6.

29. Okamura Y, Yokoi H. Development of a point-of-care assay system for measurement of presepsin (SCD14-ST). Clin Chim Acta. 2011;412:2157-61. 
30. Dellinger RP, Levy MM, Rhodes A, Annane D, Gerlach H, Opal SM, et al. Surviving sepsis campaign: international guidelines for management of severe sepsis and septic shock: 2012. Crit Care Med. 2013;41:580-637.

31. Julián-Jiménez A, Candel-González FJ, González Del Castillo J. Usefulness of inflammation and infection biomarkers in the Emergency Department. Enferm Infecc Microbiol Clin. 2014;32:177-90.

32. Kibe S, Adams K, Barlow G. Diagnostic and prognostic biomarkers of sepsis in critical care. J Antimicrob Chemother. 2011:66:ii33-40.

33. Tang BMP, Eslick GD, Craig JC, McLean AS. Accuracy of procalcitonin for sepsis diagnosis in critically ill patients: systematic review and meta-analysis. Lancet Infect Dis. 2007;7:210-7.

34. Wacker C, Prkno A, Brunkhorst FM, Schlattmann P. Procalcitonin as a diagnostic marker for sepsis: a systematic review and meta-analysis. Lancet Infect Dis. 2013;13:426-35

35. Yaegashi Y, Shirakawa K, Sato N, Suzuki Y, Kojika M, Imai S, et al. Evaluation of a newly identified solubleCD14 subtype as a marker for sepsis. J Infect Chemother. 2005:11:234-8.

36. Wu Y, Wang F, Fan X, Bao R, Bo L, Li J, et al. Accuracy of plasma sTREM-1 for sepsis diagnosis in systemic inflammatory patients: a systematic review and meta-analysis. Crit Care. 2012;16:R229.

37. Dellinger RP, Levy MM, Carlet JM, Bion J, Parker MM, Jaeschke R, et al. Surviving Sepsis Campaign: international guidelines for management of severe sepsis and septic shock: 2008. Crit Care Med. 2008:36:296-327.

38. Shirakawa K, Naitou K, Hirose J, Takahashi T, Furusako S. Presepsin (SCD14-ST): development and evaluation of one-step ELISA with a new standard that is similar to the form of presepsin in septic patients. Clin Chem Lab Med. 2011:49:937-9.

39. Hajian-Tilaki KO, Hanley JA, Joseph L, Collet JP. A comparison of parametric and nonparametric approaches to ROC analysis of quantitative diagnostic tests. Med Decis Making. 1997;17:94-102.

40. Ferrer R, Artigas A, Levy MM, Blanco J, González-Díaz G, Garnacho-Montero J, et al. Improvement in process of care and outcome after a multicenter severe sepsis educational program in Spain. JAMA. 2008;299:2294-303.

41. Kiers HD, Griesdale DE, Litchfield A, Reynolds S, Gibney RT, Chittock D, et al. Effect of early achievement of physiologic resuscitation goals in septic patients admitted from the ward on the kidneys. J Crit Care. 2010;24:563-9.

\section{Submit your next manuscript to BioMed Central and take full advantage of:}

- Convenient online submission

- Thorough peer review

- No space constraints or color figure charges

- Immediate publication on acceptance

- Inclusion in PubMed, CAS, Scopus and Google Scholar

- Research which is freely available for redistribution 